\title{
El sinjugar laborioso del juego: la otra mirada*
}

\author{
Robinson Meneses Llanos* \\ Carlos Ignacio Zúñiga López"**
}

Recibido: 10 de octubre de 2019 • Aceptado: 5 de febrero de 2020

\section{Resumen}

La reflexión propuesta a continuación emerge de la investigación realizada con la comunidad indígena Misak de la zona Guambía y Quisgó en el Municipio de Silvia, Cauca. El objetivo general consistió en comprender los sentidos de la cosmovisión en la comunidad Misak frente a los juegos ancestrales que transitan en la educación de sus niños y niñas. En un primer momento, se aborda el juego como referente temático fundamentado en la descripción breve del estado del arte y su incidencia en las otras formas de comprender que este objeto de estudio va más allá de la conceptualización lúdica; y en segundo momento, se pone en tensión la forma como el juego se convierte en una herramienta que posibilita las estrategias de aprendizaje

\footnotetext{
" Artículo de investigación, con financiación propia.
}

Citar como: Meneses, R. y Zúñiga, C. (2020). El sinjugar laborioso del juego: la otra mirada. Revista de Investigación Cuerpo, Cultura y Movimiento, 10(1), 51-67. DoI: https://doi.org/10.15332/2422474x/5961

** Doctor (c) en Educación, magíster en Educación y Desarrollo Humano y especialista en Lúdica Educativa y Entrenamiento Deportivo. Profesor de tiempo completo de la Universidad del Cauca. Integrante del grupo de investigación Kon-moción y mentor del semillero de investigación HICZO. Correo electrónico: robinsonmeneses@unicauca.edu.co; ORCID: https://orcid. org/0000-0002-9516-9647

** Magíster en Educación, línea educación multicultural y étnoeducación. Especialista en Ciencias de la Actividad Física y el Deporte. Especialista en Educación y Discapacidad. Profesor tiempo completo de la Universidad del Cauca. Integrante del grupo de investigación Salud y Motricidad de la Universidad del Cauca. Investigador en el semillero de Investigación HICZO. Correo electrónico: cizuniga@unicauca.edu.co. ORCID: https://orcid.org/0000-0002-7139-9405 
en la comunidad. La propuesta metodológica utilizada se encuentra ubicada en un enfoque cualitativo, con un diseño etnográfico, que permitió hacer del estudio cultural algo diferente a uno mismo. Los hallazgos encontrados ubican el juego en la comunidad como el sin jugar laborioso, en tanto no se juega, sino que se enseña a trabajar en las labores culturales y sociales de la familia y, así, garantizar la preservación de sus tradiciones.

Palabras clave: cultura, diversión, actitud lúdica, trabajo, tradición. 


\section{The laborious no-play of the game: The other look}

\section{Abstract}

The reflection proposed below emerges from the research carried out with the Misak indigenous community in the Guambía and Quisgó area in the Municipality of Silvia, Cauca. The overall goal was to understand the senses of the worldview of the Misak community with regards to the ancestral games that reside in the education of their children. At first, the game is approached as a thematic reference based on a brief description of the state of the art and its impact on the other ways of understanding that this object of study goes beyond the ludic conceptualization and, later, it shows the way in which the game becomes a tool that enables learning strategies in the community. The methodological proposal used is a qualitative approach, with an ethnographic design that allowed making the cultural study something different from oneself. The findings place the game in the community as laborious no-play, in so far as there is no playing, instead it is taught to work in the cultural and social tasks of the family and thus ensure the preservation of their traditions.

Keywords: culture, amusement, ludic attitude, work, tradition. 


\section{Introducción}

La noción de juego que para este escrito se pretende reflexionar está orientada a la comprensión de las actividades jugadas no desde una postura lúdica, donde siempre se conceptualiza, sino desde las tensiones que enmarcan el concepto como posibilidad de aprendizaje y la influencia que este tiene en los procesos culturales. Esto refiere quizás, a una nueva manera de ver la realidad y de vivir las relaciones sociales de la comunidad desde una visión holística, que enriquece los procesos de enseñabilidad y educabilidad, pero, sobre todo, que un entramado entre la teoría y la práctica del juego. Por ello, se ha planteado la pregunta de investigación: ¿cuáles son los sentidos otorgados por la comunidad Misak al juego? Esta postulación tiene el propósito de pensar el juego desde las posturas críticas y reflexivas que ponen en tensión las otras miradas, que se aprecian del juego como un problema serio que convoca a numerosos y destacados investigadores de distintas disciplinas científicas a otorgarle la importancia que tiene en el desarrollo de la humanidad (Toro, 2006).

Desde esta perspectiva, en clave de problema, el juego no solo es una actividad sin sentido y significado para quien la ejecuta, lo cual ha llevado a considerárselo como la pérdida de tiempo, actividad casi prohibida para el siglo xxi. Con cada vez menos tiempo para jugar en las instituciones y en la familia, se puede interpretar una sociedad disciplinaria en la producción para el consumo, que repite esquemas sociales dominantes de búsqueda de los más capaces. En otras palabras, el juego, como recurso de aprendizaje, ha perdido la posibilidad de entender y transferir ideas, valores lenguajes, modelos de conducta (Waichman, 2005, p. 55), que posibilitan estructuras de libertad en el uso del tiempo libre.

Bajo esta inquietud, este trabajo centra su atención en la comprensión del juego como objetivo inicial, teniendo en cuenta que para el Misak el perfeccionamiento físico y de conocimiento del niño y la niña se provee de acuerdo con las prácticas culturales que se desarrollan en la comunidad para lograr la armonía en su formación. Con lo anterior, esta reflexión es importante porque permite visibilizar la forma en que el juego toma otros significados que no siempre están ubicados en las prácticas lúdicas como la conciben los agentes externos a la comunidad. Es innovador porque reconoce, desde otra cultura, nuevas formas de entendimiento que permiten avanzar en el logro del bienestar de los niños en referencia al juego como proceso de interacción social 
y cultural. Ahora bien, es pertinente porque se busca describir las prácticas jugadas de los niños Misak en su contexto natural.

Como antecedente internacional, Alonqueo et al. (2017), en su investigación "Juegos escolares mapuche en el patio de recreo en una escuela rural”, desarrollada en Temuco (Chile), presenta la escuela como un espacio de socialización fundamental en el que se desarrollan variadas interacciones sociales, dentro de las que se destacan las que ocurren en el patio de recreo. El objetivo general de dicha investigación fue describir las características del juego en el patio de recreo de niños mapuches pertenecientes a una escuela rural de la comuna de Freire, en la región de La Araucanía. Participaron en este estudio 16 niños entre 6 y 12 años. Se utilizó un diseño basado en un enfoque etnográfico, usando la observación abierta para registrar seis episodios de recreo. Los resultados muestran que el tipo de juego observado es principalmente no competitivo y de ejercicio, de modo que predomina la interacción grupal entre niños de distintas edades, aun cuando se observó que niños y niñas tienden a ignorarse. Se discuten estos datos con base en el concepto de constelaciones de prácticas culturales y se identifican características propias de la cultura mapuche.

A nivel regional, se encuentra la investigación realizada por Ortiz y Gutiérrez (2016). En ella, el juego es una expresión de la cultura y la comunicación. Su objetivo general consistió comprender las características asociadas al juego tradicional desde la perspectiva de los niños, adultos y adultos mayores de la comunidad afrodescendiente. Utilizó un enfoque cualitativo, con un diseño desde la etnografía reflexiva propuesta por Murcia y Jaramillo (2000). Dentro de los principales resultados, Ortiz y Gutiérrez presentan diferentes tópicos de estudio, a saber: "características de los juegos tradicionales", "el juego en el tiempo y el tiempo en el juego" y "¿quién pone las normas y reglas del juego?”. Concluyeron que, para las tres segmentaciones poblacionales, el juego ha sido un elemento importante que se ha practicado desde hace mucho tiempo y que este mantiene su esencia en torno a sus características y espacios.

En este marco de antecedentes ha de considerarse que los juegos tradicionales y culturales permiten encontrar en sus diferentes formas, prácticas investigativas que reflexionan como el juego favorece las emocionalidades de los sujetos estudiados — generalmente en niños-, entre las que siempre resaltan el placer, la diversión, el gozo. Estos fenómenos autotélicos permiten comprender el juego desde una noción psicológica que deja de lado lo 
antropológico, lo sociológico, lo filosófico de la comunicación, la política, la norma, el civismo, y que quizás solo discurre su lenguaje en lo motriz y el aprendizaje como fundamento básico de análisis.

El seno de los debates del campo del juego ha visto prosperar trabajos concretos destinados a demostrar que jugar es un propósito tan noble como el de aprender. Sin lugar a duda, la inspiración está en los límites de la razón que nos convierte en seres inesperados; en otras palabras, las emociones también están en el alma de nuestros abuelos y animales, y, en situaciones de elevado miedo o necesidad de supervivencia, la razón se puede bloquear hasta convertirse en simples reflejos de bestia (Parra, 2012).

Pareciera por todo lo anterior que en el juego se encuentran innegables características que lo constituyen en una acción humana, entendiendo por acción toda reflexión sobre "la unidad del comportamiento humano que está sostenida por el movimiento intencionado, que puede ser definida como intencionalidad en despliegue. Es decir, ocurre a partir de un determinado sentido a expresar y conseguir" (Toro y Valenzuela, 2012, p. 227). Es decir que ocurrirá en y a partir de un determinado propósito por conseguir según el contexto, la intencionalidad, la organización, que deben estar mediatizados por los fines y objetivos que se persiguen. Lo anterior operacionaliza la acción en tanto es siempre humana e indica un proceso que se desarrolla en el tiempo en vista de un fin determinado anticipadamente (Meneses y Torres, 2010).

De acuerdo con este contexto discursivo, "es preciso ante todo localizar las superficies primeras de su emergencia" (Foucault, 2010, p. 58), en otras palabras, reconocer la génesis que sobre el juego los teóricos asumen como verdades y para ello. Bernabeu y Goldstein (2012) proponen retomar a los clásicos como Huizinga (1968), quien define elementos importantes como referente teórico de las manifestaciones lúdicas y que toma postura sobre el juego como una acción que se desarrolla en espacios y tiempos determinados. Caillois (1986), en la misma línea de Huizinga (1968), propone una clasificación de los juegos articulados también a los procesos lúdicos, que involucran el azar, la competencia, el simulacro y el vértigo.

Por su parte, Piaget (1986) admite el juego como una de las más significativas expresiones del pensamiento infantil; articula la lúdica en las nuevas estructuras mentales que posibilitan el desarrollo del niño, y las clasifica según las etapas evolutivas: juego motor y de ejercicio, juegos simbólicos y juegos de reglas. Vygotski (1988) señala dos características transicionales 
y definitorias del juego como actividad lúdica: la situación imaginaria, y la presencia de reglas ligadas también a la evolución de niño en su desarrollo evolutivo. Además, propone tres clases de juegos, el primero tiene que ver con objetos, el segundo con los juegos constructivos -mal interpretados por algunos académicos- y los juegos reglados.

Con lo anterior, se observa que estos planos de emergencia ubican el juego en la esfera de la lúdica, como un destino incierto, como algo que no sirve para nada (Bernabeu y Goldstein, 2012) o, como afirmara Huizinga (1968), "el juego está fuera de la disyunción, sensatez y necesidad; pero fuera también del contraste verdad y falsedad, bondad y maldad" (p. 19).

Este enunciado pertenece a una formación discursiva, que hay que poner en cuestión dadas las características de la sociedad que estamos viviendo. Por ello, es necesario ampliar el campo de saber del juego a través de los otros dos requerimientos que propone Foucault (2010): el que tiene que ver con describir ciertas instancias de delimitación del juego, que no se ha realizado en profundidad, debido a la confusión que existe entre juego y lúdica, y el que está relacionado con analizar finalmente las rejillas de especificación, que pondría en tensión lo que se opone, separa, reagrupa o clasifica el juego como objeto de análisis - trabajo arduo, que corresponde a los agentes del campo del discurso realizar, con miras a evolucionar en profundidad en el concepto y tomar postura frente a los teóricos tradicionales que tan mal se han interpretado-. Este argumento concuerda con la reflexión propia de la investigación: hay que tomar una postura crítica reflexiva frente a la construcción teórica del juego y posibilitar otras miradas.

Sin embargo, hay autores que se oponen a esta concepción. Sarlé (2012) propone el juego desde la educación infantil como fenómeno de aprendizaje escolar; Bernabeu y Goldstein (2012) ubican el juego como una herramienta de la creatividad y el aprendizaje; Echeita (2014) ubica el concepto en la inclusión para la no exclusión. Ahora bien, Paredes (2013) hace un recorrido conceptual sobre el origen del juego y lo propone como teoría de la lúdica desde las lenguas semánticas. Entre tanto, Jiménez (1998) conceptualiza el juego desde la postura pedagógica y lo articula con las emociones e inteligencias como base de la creatividad y la lúdica. Por su parte, Borda y Páez (2011) elaboran su conceptualización desde la psicología y proponen el juego indirectamente como herramienta creativa de aprendizaje en la escuela. Zabalza (2012), discurre su propuesta en los procesos didácticos de la educación infantil en 
la escuela como modelo integrador. Díaz (2006), por su parte, ubica el juego desde la función lúdica del sujeto y hace una interpretación teórica de la lúdica en la transformación de las prácticas pedagógicas.

En líneas de tensión crítica sobre el campo del juego y su incidencia en posturas posmodernistas, se encuentran Brailovsky (2011), Holzchuher (2012), Velásquez (2014), Montes y Castro (2015), Garlero (2018), Osorio (2019), Molina, Hurtado, Tabares y Franco (2019), entre otros. Desde sus ideas, se permite visibilizar el juego no como la lúdica, sino como un campo que ha avanzado, mostrando los desarrollos conceptuales en América Latina frente a enfoques diversos como el social, el económico y el político, y epistemológicamente ha situado otros discursos frente a las realidades sociales vividas.

Ahora bien, el contexto social y cultural donde se desarrolló la investigación se construyó a la luz la cosmovisión del pueblo Misak (Consejo Regional Indígena del Cauca [CRIC], 2019), conocido de manera generalizada como pueblo Guámbiano, aunque ellos se autorreconocido como pueblo Misak, denominación que también se asumirá para esta reflexión. Esta comunidad se localiza especialmente en el departamento del Cauca, en los resguardos indígenas ubicados en los municipios de Silvia y de Jambaló, Totoró, Caldono y Toribío. Las áreas de mayor densidad poblacional son las de los resguardos de Guambía y Quisgó en el municipio de Silvia; expansión causada por la estrechez territorial, motivada, entre otros aspectos, por la presencia de grupos al margen de la ley y de otros grupos étnicos que han hecho que el pueblo Misak se haya empezado a dispersar hacia otros territorios, conformando así el nupirau (gran territorio).

La comunidad Misak, para conservar su identidad étnica y cultura, se desenvuelve fundamentalmente alrededor de cuatro aspectos: 1) la lengua materna (namtrik) y el pensamiento, donde se desarrolla la cosmovisión y la espiritualidad guambiana, trasmitida por la oralidad de los mayores; 2) El territorio, espacio armónico entre el hombre y la naturaleza; 3) las costumbres, el constante vivir en la relación entre el hombre y la naturaleza, donde aparecen formas de vida y de vivencia que son materializadas en la minga, la danza, el vestido, la música, las creencias y la medicina, que son actos de la cotidianidad que recrean y crean la cultura; 4) la autoridad, que nace en la familia alrededor del fogón $(t u l p a)$ como pilar de la comunidad a través de los taitas y mamas, y que se hace realidad en los cabildos como eje que formula la política pública de la comunidad (CRIC, 2019). 


\section{Metodología}

La investigación se sitúo en una perspectiva cualitativa, el diseño investigativo del estudio se realizó con base en la etnografía propuesta por Cifuentes (2011). El plan de análisis se realizó según los procedimientos recomendados por la teoría fundamentada de Strauss y Corbin (2002), utilizando la codificación como un modo sistemático de reducción y refinamiento para la interpretación de los datos. Se consolidaron 4 diarios de campo, 12 entrevistas y 8 historias orales y de vida, recogidos en las mingas de pensamiento, a través de las cuales se expresan los conocimientos tradicionales, las prácticas culturales y las concepciones del pueblo Misak. Luego, se procedió a codificar los relatos como unidad de análisis a través del método de comparación constante, desde la técnica de codificación por líneas. El código se presenta entre paréntesis después de cada relato; en él mismo, se identifica la información del informante con una I, acompañada de las letras minúsculas que representan el nombre del informante, guardando su identidad; luego, se expone la técnica Dc-Et, que significan diario de campo o entrevista respectivamente, cuando se recogió la información, $\mathrm{y}$, por último, se ubica el relato $(\mathrm{R})$ con el respectivo número consecutivo que identifica el relato. Esto permitió la agrupación con otros relatos con características semejantes y hacer agrupaciones desde las categorías más simples o abiertas, pasando por las axiales o conceptuales, hasta obtener las categorías selectivas. Posteriormente, se hizo un acercamiento más detallado con informantes claves - Taitas y Mamas ( 3 hombres, 1 mujer)-, de los cuales se obtuvo una entrevista semiestructurada y se finalizó con las historias orales y de vida para finalmente obtener la categoría central.

Frente a los aspectos éticos, se solicitaron los permisos correspondientes para avalar la ejecución del proyecto. Se protegió la identidad de los informantes a través de la asignación de códigos personales para mantener su anonimato y en los procesos de recolección, procesamiento y análisis de los datos se manejaron con respeto y de manera confidencial.

\section{Resultados}

Una vez recogida la información, esta se analizó y emergieron cuatro temáticas, descritas en forma de acciones jugadas que posibilitan comprender los sentidos otorgados por la comunidad a estas prácticas. 


\section{Actividad de hombres a punta de golpes: el "zarambico"}

Cuentan los mayores, mayoras, sabedores y sabedoras de la comunidad que cuando el $n u$-Misak —se refiere al bebe niño o niña recién nacido, o también un muñeco o muñeca de trapo hecho con recortes de tela que representa a los niños Misak que permite el autorreconocimiento— llega (nace), comienza a aprender con su familia alrededor del fogón de leña. En estos procesos educativos son las mujeres las que se encargan de proyectar la educación a las niñas y los hombres a los niños, quienes después de cumplir los tres (3) años de vivenciar lo que su taita o mayor hace él quiere repetir las mismas costumbres, como la de hacer bailar el Zarambico, como lo hace su papá o los mayores.

Pero ¿qué es el Zarambico? Dicen los taitas que es un juego que realizan solo los hombres, con el objetivo de que el niño tenga contacto con la tierra y su relación con el cosmos y su parte natural; este tiene su forma de línea vertical, que en la simbología Misak pertenece a los hombres. Es hecho de madera fina y solo debe ser cosechado en la noche entre las nueve y las horas de la madrugada del día siguiente, porque esas son las horas en que el espíritu se conecta fuertemente con el árbol y así la madera es mucho más fina y no se daña tan rápido.

El niño juega con el primer zarambico, que es elaborado por el papá quien prepara la madera que debe estar seca. El zarambico debe tener dos piezas; una es el trozo de madera hecha en forma cónica con la ayuda de un machete o peinilla, "parecido a un trompo, pero sin cabeza" (I: jp/Dc:03/R:21), dice el taita. El otro segmento está compuesto por un "palito" delgado del largo según la estatura de la persona que lo va a jugar, sin importar el tipo de madera. Lleva amarrado en su punta un pedazo de cabuya trenzada, del largo que garantice poder golpear el madero al momento de hacerlo bailar sin tener que agacharse demasiado. A este segmento se le denomina Zurriago.

La simbología del juego se desenvuelve con la representación del inicio y el final de los tiempos en la cosmovisión Misak. Inicialmente, se desarrolla envolviendo la cabuya del zurriago en la parte superior del zarambico; después de esto se coloca en el suelo envuelto, lo cual da inicio a la segunda etapa, que consiste en lanzar o halar el zurriago hacia adelante o al frente de manera tal que, al hacerlo, el zarambico se mueva, baile o gire en su punta, con los golpes que se dan con el zurriago para que no deje de girar y suene lo suficiente. Este sonido representa los sonidos de la madre tierra y el contacto 
del zarambico, desde el momento de su contacto con el zurriago, tiene la simbología del fin. El principio y el fin están representados por las dos espirales, "porque cuando se envuelve es hacia un lado y cuando se gira es al otro lado" (I:ac/Dc:03/R:18). Todo esto tiene relación con el lenguaje natural, ya que está directamente relacionado con la tierra y los ciclos de la vida.

Como todo proceso educativo, el niño aprende a jugar con el zarambico con el paso de los años, en la compañía del mayor o taita, quien le indica cómo ubicar, envolver, lanzar, y "cuetear" — acción de pegarle con el zurriago-el madero. Así, ellos aprenden la posición básica para jugar: con los pies separados al ancho de los hombros, uno más adelante que el otro, dependiendo de la mano con la que se coge el zurriago; las rodillas deben estar semiflexionadas para garantizar un buen golpe del zurriago al madero al momento de golpear el zarambico; el tronco debe estar flexionado adelante con el objetivo de tener un acercamiento frontal al madero; los brazos se ubican abiertos. El que maneja el zurriago hace un movimiento lateral para utilizarlo como látigo para golpear y el otro cumple dos tareas, la primera es de balance del cuerpo y la segunda, la de poder sostener el "ruana" "bufanda" para que no se complique cuando se juega. Cuando el niño sabe jugar, ya está en la edad de más o menos 10 años, cuando ya puede manipular las herramientas sin el riesgo de hacerse daño para la elaboración de su zarambico.

\section{El canyico para un mandado: se cuelga el encargo en el timón}

El carrito es un implemento que está elaborado con una vara de madera larga, simulando una carretilla de un solo brazo. Se compone de tres partes: ruedas, chasis y timón. La primera es muy importante para el implemento, porque el juguete no tendría la función de desplazamiento de forma divertida. Este rodillo es elaborado (con cuchillo o machete) en un trozo de madero muy redondo, para garantizar que gire al momento de empujarlo. Se le tala una muesca en el centro, a su alrededor, donde encaja el segundo componente, la vara larga, que en uno de sus extremos finaliza con una "horqueta" (horquilla) con la cual se ensambla con el rodillo de madera. El tercer componente es un segmento de vara que hace las veces de timón y que se ubica en la porción distal de la vara. La distancia donde se coloca este timo depende de la estatura del niño, pues, según este aspecto, da mayor comodidad para maniobrar el carrito. Este timón se sujeta a la vara central mediante el empleo de un clavo o a través de un amarre. 
Consiste en que el niño o la niña coge el timón con las dos manos apoyando el chasis o vara en uno de sus hombros y garantiza que la "horquilla" quede bien ubicada en la muesca del rodillo. Cuando esto sucede, el niño puede empujar el juguete de tal manera que este ruede desplazando el carrito por el espacio moviendo el timón a la derecha o izquierda según la necesidad para esquivar los obstáculos. Cuando es requerido para un mandado, el niño cuelga el encargo en el timón o sobre un clavo adicional que se ubica más debajo de este.

Mediante la elaboración de este artefacto de la vida cotidiana, los mayores o mayoras, o casukos - jóvenes Misak con autoridad sobre el niño- le enseñan las "diferentes virtudes y propiedades del árbol con el cual elabora el instrumento para que él aprenda a trabajar en el mandado" (I:md/Et:01/R:27). Por otra parte, se les enseña "las formas de elaboración y el uso adecuado de las herramientas de trabajo" (I:md/Et:01/R:22). Con el empleo del carrito también se pueden manejar los conceptos de espacialidad, así como los de tiempo y cantidad.

El niño, mediante el empleo del carrito, fortalece su proceso relacional, inicialmente con su padre como también con los vecinos y con sus amigos de la vereda o la escuela.

\section{El juego del aro Nanrrí: entre golpear y mover la cintura}

Este implemento es elaborado con un "bejuco" especial, llamado por la comunidad Misak como chiyax (I:md/Et:03/R:15). Este es enrollado dos o tres veces de forma entretejida para darle forma al aro. Adicionalmente, se elabora con un trozo de madera un pequeño bastón para direccionar el aro. El juego consiste en hacer rodar el aro por el piso, dándole impulso mediante el uso del bastón. Para esto, se golpea consecutivamente al aro, de modo que este pueda rodar más durante su desplazamiento sobre el piso, siendo este su objetivo final del juego. El niño debe mantenerlo durante el mayor tiempo posible sin que se pierda el control del aro.

Cuando el aro es utilizado por las niñas el objetivo del juego es diferente, pues ellas lo que buscan es hacerlo girar alrededor de su cintura o de sus brazos, mediante movimientos cadenciosos de estos segmentos corporales. 


\section{Dos espíritus importantes para \\ la comunidad: entre el viento y el aguacero}

Para este juego se emplea como herramienta didáctica "el caballo", un juguete elaborado en dos partes: un trozo de madera (vara), el cual constituye el cuerpo del animal, y la cabeza del caballo, elaborada en diferentes materiales; por ejemplo, cuero de animales, trozos de madera o retazos de tela con la que se elabora parte del cuerpo del equino. En algunas ocasiones, a este juguete le adicionan un par de correas para simular la rienda con la cual se le da manejo al implemento.

El juego consiste en que la mayora, montada en su caballo y seguida por los niños y sus madres, simulan ser la lluvia (mamá Dominga) mientras atrás, el mayor asumiendo el rol de viento (taita Ciro) los persigue haciendo ruidos alrededor de la casa, que en este juego es la representación de la montaña. Además, la casa para los Misak es el sitio donde la comunidad puede entrar para protegerse de la lluvia o del viento. Para el proceso de enseñanza-aprendizaje sobre el viento, como un elemento esencial en la vida en los Misak, la mayora explica sobre el origen, dirección, efectos y causas del viento, “por eso es importante para esta comunidad la ubicación de la casa sobre el terreno donde se construye" (I:ac/Dc:01/R:8). Le dan importancia al lugar sobre el cual se colocara la puerta de esta, para así evitar algunos efectos del viento; expresan además la importancia de la cocina como factor importante para proteger la casa contra la acción del viento. Por otra parte, se hace referencia a algunos hechos simbólicos del viento, por ejemplo: "Como este se roba los sobreros, levanta los anacos entre otras prendas de vestir, además trae la lluvia y en ocasiones se llevaba las nubes para impedir que esta llegue" (I:ac/ Dc:03/R:11). También reflexionan sobre los tiempos de convivencia entre el viento y el aguacero, dentro del cual la lluvia llega a irrigar sus tierras.

Para los Misak, el viento es femenino y, por eso, ellos lo denominan mama Dominga; el aguacero es considerado masculino y, por esta razón, lo llaman taita Ciro, y representan dos espíritus importantes para la comunidad. Esta actividad representa las relaciones interpersonales entre niños y niñas, padres y madres, casukos y tías, ya que no hay separación por géneros, como suele suceder en otros juegos. Aquí los niños y las niñas comparten de forma directa la actividad de socialización de la historia y el juego del viento. 


\section{Discusión}

La comprensión de las actividades jugadas no desde una postura lúdica, sino desde las tensiones que enmarcan el concepto de juego como posibilidad de aprendizaje y su influencia en los procesos culturales representados en la precepción del mundo que envuelve la comunidad Misak, permitió desde las acciones jugadas, el Zarambico, el Canyico, el Nanrí y el Nu-Misak, representar las evidencias de su oralidad tradicional, para lograr perpetuar su tradición cultural, social y de cosmovisión, de una generación a otra. Con respecto a este tema, Le Breton (2007) plantea que la percepción del mundo se posibilita a través de los cinco sentidos, y es el resultado de una educación culturalmente codificada y de una experiencia personal única. Una sociedad define maneras particulares para establecer selecciones planteando entre ella y el mundo el tamizado de los significados de los valores y procura de cada uno de ellos las orientaciones para existir en el mundo y comunicarse con el entorno.

Con lo anterior, cada pueblo indígena posee una identidad cultural, estrechamente asociada a ciertas tradiciones y a su dialecto, que es lo que se destaca en este informe; sin embargo los sentidos que le otorgan los mayores y mayoras a las acciones jugadas rompe con la tradiciones de otros pueblos del juego por el juego y lo ubican en las acciones jugadas para sembrar en sus niños la cultura. Esta tradición se ubica en lo que Orduna (2003) denomina a dicho conjunto de rasgos y elementos que distinguen a cada comunidad como identidad cultural, por la cual la comunidad es juzgada, valorada, apreciada, definida o rechazada por otros y por la misma comunidad.

\section{Conclusiones}

Los sentidos que la comunidad otorgó al juego permitieron comprender que estos se ubican en el desarrollo de su cosmovisión, el aprendizaje por el trabajo y respeto por la naturaleza, categorías conceptuales desarrolladas en los hallazgos.

Para los Misak el jugar laborioso es una acción vivida que permite comprender la relación niño, cultura, naturaleza y familia, a través de juguetes que permiten el acercamiento de los infantes con su comunidad. Esta trasciende el juego por el aprendizaje colaborativo, comunal y cultural en 
pos del sostenimiento de su cosmovisión, en tanto el juego no se juega como una actividad infantil cotidiana, sino que se manifiesta en la relación de la enseñanza con las labores de las mingas culturales y sociales de la familia, lo que garantiza la preservación de sus tradiciones. De esta manera, estos garantizan, también, en los niños el desarrollo del proceso cognitivo, físico y desarrollo socioafectivo.

Las acciones laboriosas jugadas cumplen con varios procesos de enseñanza-aprendizaje entre los niños, niñas y la comunidad, que son: 1) perpetuar todas sus raíces culturales; 2) todos los juguetes dentro del juego tienen la función de proceso didáctico, para poder hacer la enseñanza cultural de una forma más fácil para las niñas y los niños; 3) que los niños aprehendan todo lo concerniente a las labores cotidianas de la comunidad; 4) los juegos están encaminados como objetivo fundamental al desarrollo, fortalecimiento o afianzamiento de las capacidades condicionales (fuerza, resistencia, velocidad y flexibilidad) y perceptivo-motrices (corporalidad, espacialidad, temporalidad, coordinación y equilibrio); 5) en cuanto a lo social, el juego para los Misak enseña la importancia del papel del hombre y el de la mujer dentro de su comunidad. Por esto, en la gran mayoría de las acciones jugadas se establece la separación de género en el proceso de enseñanza aprendizaje, según el contexto en el que se desenvuelve la comunidad.

\section{Referencias}

Alonqueo, P., Loncón, M., Vásquez, F., Gutiérrez, J. y Parada, L. (2017). Juegos escolares mapuche en el patio de recreo en una escuela rural. Revista de Psicología, 26(2), 1-15. DOI: http:dx.doi.org/10.5354/0719-0581.2017.47957

Bernabeu, N. y Goldstein, A. (2012). Creatividad y aprendizaje. Bogotá: Ediciones de la U.

Borda, E. y Páez, E. (2011). Ayudas educativas. Creatividad y aprendizaje. Bogotá: Cooperativa Editorial Magisterio.

Brailovsky, D. (2011). El juego y la clase: ensayos críticos sobre la enseñanza posttradicional. Buenos Aires: Centro de Publicaciones Educativas y Material Didácticos S.R.L.

Caillois, R. (1994). Los juegos y los hombres. Bogotá: FCE. 
Cifuentes, R. (2011). Diseño de proyectos de investigación cualitativa. Buenos Aires: Ediciones Novedades Educativas.

Consejo Regional Indígena del Cauca. (2019, 23 de octubre). El Consejo Regional Indígena del Cauca. Descripción contexto y características propias de la cultura Misak. Recuperado de: https://www.cric-colombia.org/portal/

Echeita, G. (2007). Educación para la inclusión o educación sin exclusiones. Bogotá: Narcea.

Foucault, M. (2010). La arqueología del saber (A. Garzón del Camino, trad.). (2. ${ }^{a}$ ed.). México: Siglo XXI.

Garlero, J. (2018). Recreación y Dictadura. Un caso en América Latina. México: Colectivo Editorial Casa de las Preguntas.

Holzchuher, C. (2012). Cómo organizar aulas inclusivas. Propuestas y estrategias para acoger las diferencias (P. Manzano, trad.). Madrid: Narcea Ediciones.

Huizinga, J. (1968). Homo luden. Buenos Aires: Emercé.

Jiménez, C. A. (1998). Pedagogía de la creatividad y de la lúdica: emociones, inteligencia y habilidades secretas. Bogotá: Cooperativa Editorial Magisterio.

Le Breton, D. (2007). El sabor del mundo. Una Antropología de los sentidos. Buenos Aires: Ediciones Nueva Visión.

Meneses, R. y Torres, L. (2010). Los sentidos de la formación práctica en los estudiantes de la Licenciatura en Educación Básica con Énfasis en Educación Física, Recreación y Deporte de la Universidad del Canca [tesis de maestría]. Universidad de Manizales. Manizales, Colombia.

Molina, V., Hurtado, A., Tabares, F. y Franco, S. (2019). Metodologías otras para el estudio del ocio y la recreación en América Latina. México: Editorial Casa de las Preguntas.

Montes, M. y Castro, M. (2015). Juego para niños con necesidades educativas especiales. México: Pax México.

Murcia, N. y Jaramillo, L. (2000). La complementariedad etnográfica. Investigación Cualitativa. Una guía posible abordar estudios sociales. Armenia: Kinesis.

Orduna, G. (2003). Desarrollo local, educación e identidad cultural. Estudios sobre Educación, 4, 67-83. Recuperado de: http://dadun.unav.edu/bitstream/10171 /8408/1/Estudios\%20Ee.pdf

Ortiz, C. y Gutiérrez, J. (2016). Los juegos tradicionales de la comunidad afrodescendiente de San Antonio y El Llanito del Salado, Municipio de Páez, Departamento del Cauca [tesis de pregrado no publicada]. Universidad del Cauca. 
Osorio, E. (2019). Asuntos presentes en el campo de la recreación. México: Colectivo Editorial Casa de las Preguntas.

Paredes. J. (2003). Juego, luego soy. Sevilla: Wanceulen Editorial Deportiva.

Parra. J. (2012). Inspiración. Asuntos intimos sobre creación y creadores. Bogotá: Cooperativa Editorial Magisterio.

Piaget, J. (1986). Psicología y pedagogía del juego. Barcelona: Agostini.

Sarlé, P. (2012). Juegos y aprendizaje escolar: los rasgos del juego en la educación infantil. Buenos Aires: Centro de Publicaciones Educativas y Materiales Didácticos.

Strauss, A. y Corbin, J. (2002). Bases de la investigación cualitativa: técnicas y procedimientos para desarrollar la teoría fundada (1. ${ }^{a}$ ed.). Medellín: Editorial Universidad de Antioquia.

Toro, S. (2006). Juego y Motricidad. Motricidad y Persona, 1.

Toro., S. y Valenzuela, P. (2012). Desde la acción a la enacción. Más allá del movimiento y de la Educación Física. Estudios Pedagógicos, 38(1), 211-230. Recuperado de: https://scielo.conicyt.cl/pdf/estped/v38nespecial/art12.pdf

Velásquez, J. (2014). El desarrollo de competencias con juegos: ambientes lúdicos de aprendizaje diseño y operación. México: Trillas.

Waichman, P. (2005). Modelos económicos, modelos... En J. F. Tabares, A. F. Ossa y V. A. Molina, (coords.), El ocio, el tiempo libre y la recreación en América Latina: problematizaciones y desafíos (pp. 47-64). Medellín: Editorial Civitas.

Vygotski, L. (1988). El desarrollo de los procesos psicológicos superiores. Barcelona: Crítica.

Zabalza, M. (2012). Didáctica de la educación infantil. Bogota: Ediciones de la U. 